\title{
Transference of Wheat Expressed Sequence Tag- Simple Sequence Repeats to Paspalum Species and Cross-Species Amplification of Paspalum notatum Simple Sequence Repeats: Potential Use in Phylogenetic Analysis and Mapping
}

\author{
Lorena Adelina Siena, María Esperanza Sartor, Camilo Luis Quarin, \\ Francisco Espinoza, and Juan Pablo A. Ortiz ${ }^{\star}$
}

\begin{abstract}
The genus Paspalum L. includes numerous species of agronomic importance. The objectives of this work were to evaluate the transferability and polymorphism of publicly available wheat (Triticum aestivum L.) expressed sequence tag (EST)-simple sequence repeat (SSR) (wEST-SSR) markers to Paspalum spp., assess the cross-species amplification of Paspalum notatum Flüggé genomic SSRs (PnSSRs) within the genus, and evaluate both types of markers for phylogenetic analyses and mapping. Thirty-two accessions including 10 species were used. Moreover, $65 F_{1}$ hybrids of $P$. notatum were used for mapping. Transferability ratio of wEST-SSRs was $72.72 \%$. On average 19.25 bands per primer combination were obtained. Cross-species amplification of PnSSRs was $55.37 \%$, with an average of 9.45 fragments per primer pair. Both types of markers differed in the amplification capacity between primers pairs and species. Clustering analysis with wEST-SSR data discriminated accessions by species and taxonomic groups. Genomic relationships were in agreement with previous works indicating that wEST-SSRs are adequate for phylogenetic surveys in the genus. Mapping experiments showed that both wEST-SSRs and PnSSRs mapped scattered in the genome. One hundred two new markers were added to the existent $P$. notatum linkage groups. Primer pairs ksum206, ksum219, and PN03 F2 generated markers linked to apospory. Sequences of EST-SSRs experimentally mapped in $P$. notatum showed 46.23 to $55.27 \%$ similarity with the original wheat EST. A preliminary comparative mapping analysis was carried combining experimental and in silico mapping results.
\end{abstract}

L.A. Siena, and J.P.A. Ortiz, Laboratorio de Biología Molecular, Facultad de Ciencias Agrarias, Universidad Nacional de Rosario (UNR), Campo Experimental Villarino s/n, CC 14, (S2125ZAA) Zavalla, Santa Fe, Argentina; M.E. Sartor, C.L. Quarin, F. Espinoza, and J.P.A. Ortiz, Instituto de Botánica del Nordeste (IBONE-CONICET), Facultad de Ciencias Agrarias, Universidad Nacional del Nordeste (UNNE), Sargento Cabral 2131, CC 209, (3400) Corrientes, Argentina. Received 28 Apr. 2013. *Corresponding author (jortiz@unr.edu.ar).

Abbreviations: ACL, apospory controlling locus; AFLP, amplified fragment length polymorphism; BLASTn, basic local alignment search tool for nucleotide sequences; cpDNA, chloroplast DNA; EDTA, ethylenediaminetetraacetic acid; EST, expressed sequence tag; ITS, internal transcribed spacer; LOD, logarithm of the odds; PAL, phenylalanine ammonia-lyase; PCR, polymerase chain reaction; PnSSR, Paspalum notatum genomic simple sequence repeat; RFLP, restriction fragment length polymorphism; SSR, simple sequence repeat; Tm, melting temperature; UPGMA, unweighted pair group method with arithmetic mean; wEST-SSR, wheat expressed sequence tag-simple sequence repeat.

$\mathrm{P}$ ASPALUM is a large genus of the Poaceae family (subfamily Panicoideae and tribe Paniceae), with about 330 species distributed mainly in tropical, subtropical, and temperate regions of the Americas (Zuloaga and Morrone, 2005). Ploidy levels vary from diploid $(2 x)$ to, exceptionally, hexadecaploid (16x) (Burton, 1940; Quarin and Lombardo, 1986) and modes of reproduction from exclusive sexuality to obligate apomixis (Quarin, 1992). About $80 \%$ of the species are polyploids, and approximately half

Published in Crop Sci. 54:240-254 (2014).

doi: 10.2135/cropsci2013.04.0275

(C) Crop Science Society of America | 5585 Guilford Rd., Madison, WI 53711 USA

All rights reserved. No part of this periodical may be reproduced or transmitted in any form or by any means, electronic or mechanical, including photocopying, recording, or any information storage and retrieval system, without permission in writing from the publisher. Permission for printing and for reprinting the material contained herein has been obtained by the publisher. 
of those are tetraploids. Apomictic reproduction is associated with polyploidy and usually, tetraploid apomictic species have diploid, self-incompatible, sexual co-specific races (Quarin, 1992).

Because of its many species and high variability, the genus Paspalum has been divided into subgenera, sections, and informal infrageneric groups. The most widely accepted infrageneric classification for North American species was proposed by Chase (1929), who recognized two subgenera: Ceresia (with eight species) and Paspalum (containing the remaining species), the latter with 25 informal subgroups. However, Chase's grouping has been repeatedly revised by subsequent classifications (Zuloaga and Morrone, 2005; Souza-Chies et al., 2006). Recently, three subgenera, Anachyris (Nees) Chase, Ceresia (Pers.) Rchb., and Paspalum (with 28 informal groups), were recognized by Zuloaga and Morrone (2005) in their revision of the austral South American species. Traditionally, phylogenetic relationships within the genus were determined by analyzing the morphology mainly of inflorescences and/ or by hybridizing selected parents and observing chromosome pairing during meiosis in the hybrids (e.g., Burson et al., 1973; Burson, 1978; Caponio and Quarin, 1987; Quarin and Norrmann, 1990). More recently, nuclear molecular markers such as restriction fragment length polymorphisms (RFLPs) (Jarret et al., 1998) and random amplified polymorphic DNAs (Miz and Souza-Chies, 2006) as well as sequence data from the nuclear ribosomal ribonucleic acid internal transcribed spacer (ITS) or the chloroplast DNA (cpDNA) $p s b A-t r n \mathrm{H}$ spacer and trnL intron have been used as tools for phylogenetic analyses (Souza-Chies et al., 2006; Essi and Souza-Chies, 2007, Giussani et al., 2009, Rua et al., 2010). However, no uniform criterion of classification has been achieved thus far (Rua et al., 2010).

The genus has an important economic value because several species are used for forage, turf, or ornamental purposes (Burson and Bennett, 1971). Paspalum atratum Swallen, Paspalum dilatatum Poir., Paspalum guenoarum Arechav., P. notatum, and Paspalum urvillei Steud. are excellent fodder crops (Zuloaga and Morrone, 2005). In particular, the diploid form $(2 n=2 x=20)$ of $P$. notatum (Pensacola bahiagrass) is one of the most important warmseason forages in the southeastern United States while the tetraploid form $(2 n=4 x=40)$ (common bahiagrass) is a principal component of natural pastures of the subtropical areas of Argentina, Brazil, and Paraguay (Gates et al., 2004). Genetic linkage maps of the tetraploid race have been developed based mainly on single-dose amplified fragment length polymorphism (AFLP) markers (Stein et al., 2007). The recombination maps cover more than $80 \%$ of the parental genotype genomes, but because of the anonymous nature of AFLP markers, comprehensive comparative analyses with model grasses was not possible. A unique genomic region characterized in detail is the chromosome segment carrying the control for apospory (apospory controlling locus [ACL]). By combining AFLP and RFLP markers, it was determined that ACL is a large chromosome segment with suppressed recombination and synteny with segments of rice (Oryza sativa L.) chromosomes 2 and 12 and maize (Zea mays L.) chromosomes 3 and 5 (Martínez et al., 2003; Stein et al., 2004; Pupilli et al., 2004; Hojsgaard et al., 2011; Podio et al., 2012).

Simple sequence repeats (SSRs) are useful markers for a variety of applications in plant genetics and breeding because of their reproducibility, multiallelic nature, codominant inheritance, relative abundance, and good genome coverage (Powell et al., 1996). However, only a few SSR markers have been developed in species of Paspalum. A total of 5, 11, and 19 SSR primer pairs were reported in Paspalum vaginatum Sw. (Liu et al., 1995), P. notatum (Cidade et al., 2009), and $P$. atratum (Cidade et al., 2010), respectively. Likewise, 12 primer pairs were developed in $P$. dilatatum and related species (Speranza and Malosetti, 2007). Expressed sequence tag (EST)-SSRs are genic microsatellites derived from transcribed DNA that contains microsatellite repeats (Varshney et al., 2005). These genic SSR markers are relatively easy and inexpensive to generate because they are a byproduct of sequence data from gene or EST libraries. A large wheat EST-SSR (wEST-SSR) collection is publicly available (http://wheat. pw.usda.gov/ITMI/EST-SSR/Cornell [accessed 6 Oct. 2011]). Wheat EST-SSRs have a high level of transferability to close wild relatives of wheat and have been used to trace chromosome segments in a wide range of grass species (Zhang et al., 2005, 2007). Moreover, wEST-SSR markers have been used for comparative mapping in wheat and as anchor loci for comparative mapping between wheat and rice (Yu et al., 2004a, 2004b).

The objectives of this work were (i) to evaluate the transferability and the level of polymorphism of publicly available wEST-SSR markers to Paspalum species, (ii) to test the cross-species amplification of a set of $P$. notatum genomic SSRs (PnSSRs) to other species of the genus, and (iii) to use both types of markers for phylogenetic analysis and mapping.

\section{MATERIALS AND METHODS Plant Materials}

Thirty-two accessions representing 10 species, nine from subgenus Paspalum from five taxonomic informal groups (Decumbentes, Dilatata, Livida, Notata, and Plicatula) and one from subgenus Anachyris, were used. Accessions were collected from natural populations located in nine different countries of the Americas or obtained experimentally. Species were chosen based on the availability of germplasm and their agronomic importance. One individual per accession was genotyped. The origin, ploidy level, and the mode of reproduction of each accession are indicated in Table 1 . In addition, $65 \mathrm{~F}_{1}$ hybrids of $P$. notatum were used for mapping experiments. These plants 
Table 1. Paspalum accessions used to evaluate the transferability and polymorphisms of wheat expressed sequence tagsimple sequence repeats and genomic Paspalum notatum simple sequence repeat markers.

\begin{tabular}{|c|c|c|c|c|c|}
\hline $\begin{array}{l}\text { Taxonomic } \\
\text { group }\end{array}$ & Species & Accession & Origin $^{\dagger}$ & $\begin{array}{l}\text { Ploidy } \\
\text { level }\end{array}$ & $\begin{array}{l}\text { Mode of } \\
\text { reproduction }\end{array}$ \\
\hline \multirow[t]{7}{*}{ Plicatula } & P. lenticulare & V11724 & BR, GO, 39 km southwest of Gaçu & $4 x$ & Apomictic \\
\hline & & N188§ & PA, 50 km northeast of Concepción way to Paso Barreto & $2 x$ & Sexual \\
\hline & P. guenoarum & Chané FCA & BO, Santa Cruz, Concepción & $4 x$ & Apomictic \\
\hline & & Baio & $\mathrm{BR}, \mathrm{RS}$, central depression region & $4 x$ & Apomictic \\
\hline & & Azulado & $\mathrm{BR}, \mathrm{RS}$, central depression region & $4 x$ & Apomictic \\
\hline & P. atratum & Cambá FCA & BR, MS, Terenos & $4 x$ & Apomictic \\
\hline & P. nicorae & MS5 & AR, Santa Fe, Cayastá & $4 x$ & Apomictic \\
\hline \multirow[t]{2}{*}{ Livida ${ }^{\ddagger}$} & P. buckleyanum & MS1 & AR, Chaco, 56 km north of Sáenz Peña. Route 95 & $5 x$ & Apomictic \\
\hline & & Q4303 & PA, 120 km west of Asunción, Transchaco route & $6 x$ & Apomictic \\
\hline \multirow[t]{7}{*}{ Dilatata $^{\ddagger}$} & P. urvillei & Q4226 & BR, RS, Restinga Seca & $4 x$ & Sexual \\
\hline & P. dilatatum common type & LS3 & AR, Santa Fe, Zavalla & $5 x$ & Apomictic \\
\hline & & $\mathrm{H} 163$ & AR, Misiones, Posadas & $5 x$ & Apomictic \\
\hline & P. dilatatum 'Virasoro' & Q2960 & AR, Corrientes, Virasoro & $4 x$ & Sexual \\
\hline & P. dilatatum 'Chirú' & Q4081 & UR, Paysandú, Molles Grande & $6 x$ & Apomictic \\
\hline & P. dilatatum 'Sexual Chirú' & $69-12$ & Experimental & $6 x$ & Sexual \\
\hline & P. dilatatum subsp. flavescens & Q3952 & UR, Montevideo & $4 x$ & Sexual \\
\hline \multirow[t]{2}{*}{ Decumbentes ${ }^{\ddagger}$} & P. unispicatum & U15 & AR, Chaco, 3 km southwest of de Campo Largo & $2 x$ & Sexual \\
\hline & & MS14 & AR, Formosa, 10 km west-northwest of Pozo del Tigre & $3-4 x$ & Apomictic \\
\hline \multirow[t]{12}{*}{ Notata $^{\ddagger}$} & P. notatum & Tif9 & Tifton, GA, United States & $2 x$ & Sexual \\
\hline & & Q4295 & AR, Santa Fe, Cayastá & $3 x$ & Apomictic \\
\hline & & Q4084 & AR, Santa Fe, Cayastá & $4 x$ & Apomictic \\
\hline & & Q4188 & Experimental & $4 x$ & Sexual \\
\hline & & Q4117 & BR, RS, unknown locality & $4 x$ & Apomictic \\
\hline & & Q3775 & Mexico, Tamaulipas & $4 x$ & Apomictic \\
\hline & & Q4181 & Cuba, Matanzas, Perico & $4 x$ & Apomictic \\
\hline & & Q4010 & BR, MS, Tres Lagos & $4 x$ & Apomictic \\
\hline & & SV2893 & PE, Cajamarca, Cajabamba, El Huayo, 2100 m asl & $4 x$ & Apomictic \\
\hline & & ST2369 & BO, Santa Cruz de la Sierra, Salinas & $4 x$ & Apomictic \\
\hline & & N160 & PA, Amambay, Amambay, $25 \mathrm{~km}$ north of Pedro Juan Caballero & $4 x$ & Apomictic \\
\hline & & Q4276 & AR, Corrientes & $4 x$ & Apomictic \\
\hline \multirow[t]{2}{*}{ Anachyris" } & P. simplex & U36 & AR, Chaco, Avia Terai & $3 x$ & Sexual \\
\hline & & Q4121 & AR, Santa Fe, 16 km south of de Alejandra & $4 x$ & Apomictic \\
\hline
\end{tabular}

${ }^{\dagger}$ AR, Argentina; BO, Bolivia; BR, Brazil; GO, State of Goiás (Goiânia); MS, State of Mato Grosso do Sul; PA, Paraguay; PE, Peru; RS, State of Rio Grande do Sul; UR, Uruguay.

\#Informal taxonomic group of the subgenus Paspalum.

§N188 was formerly classified as Paspalum limbatum (Espinoza et al., 2001). Paspalum limbatum Henrard is now considered to be a synonym of Paspalum lenticulare Kunth (Oliveira and Valls 2008).

"Subgenus (Zuloaga and Morrone 2005).

were the remnants of a larger mapping population segregating for the mode of reproduction (aposporous vs. nonaposporous) used to construct a genetic map of the species and localize the ACL (Stein et al., 2004, 2007). All material belonged to the live germplasm collection of the Instituto de Botánica del Nordeste, Corrientes, Argentina. Hexaploid wheat cultivar Federal was used as an amplification control and outgroup.

\section{Molecular Marker Assays}

The DNA extraction was performed using the cetyltrimethylammonium bromide method according to Martínez et al. (2003). A group of $55 \mathrm{wEST}-\mathrm{SSR}$ markers publicly available at http://wheat.pw.usda.gov/ITMI/EST-SSR/Cornell (accessed 6 Oct. 2011) was used (Supplemental Table S1). Informative markers were screened by amplifying wEST-SSRs from the tetraploid P. notatum genotypes Q4188 and Q4117. Polymerase chain reaction (PCR) conditions were initially as described by $\mathrm{Yu}$ et al. (2004b). In cases where amplification failed, a touchdown protocol was used (Don et al., 1991): an initial denaturation step of $94^{\circ} \mathrm{C}$ for $1 \mathrm{~min}$ was followed by 10 cycles in which the initial annealing temperature (range $55-65^{\circ} \mathrm{C}$ ) decreased $0.5^{\circ} \mathrm{C}$ per cycle to a "touchdown" temperature (range $50-60^{\circ} \mathrm{C}$ ) followed by 35 cycles of amplification and a final elongation at $72^{\circ} \mathrm{C}$ for $10 \mathrm{~min}$. The melting temperature (Tm) of each primer pair was calculated according to the formula $[4 \times$ number of $(\mathrm{G}+\mathrm{C})+2 \times$ number of $(\mathrm{A}+\mathrm{T})]$ (AccessQuick RT-PCR System; Promega). Based on this value, four annealing temperatures per primer pair were tested (from $4^{\circ} \mathrm{C}$ above to $2^{\circ} \mathrm{C}$ below $\mathrm{Tm}$ ). Reactions were performed in a Gene Pro TC-E96G gradient thermal cycler (BIOER). The optimal Tm was considered the highest temperature that produced reproducible amplification in at least two independent reactions. 
Eleven primer pairs amplifying $P$. notatum genomic SSR markers were also analyzed (Cidade et al., 2009) (Supplemental Table S2). Amplification reactions were performed as described by Cidade et al. (2009) in a Personal Thermal Cycler MyCycler (Bio-Rad). All PCR products were electrophoresed in 5\% $(\mathrm{w} / \mathrm{v})$ polyacrylamide in $1 \mathrm{x}$ Tris, borate, and ethylenediaminetetraacetic acid (EDTA) buffer denaturing gels at $60 \mathrm{~W}$ for $1 \mathrm{~h}$, fixed in $10 \%$ (v/v) $\mathrm{CH}_{3} \mathrm{COOH}$ (acetic acid), and silver stained using the DNA Silver Stain System (Promega) as described by Stein et al. (2004). Only clear bands of the predicted molecular weight were scored.

\section{Data Analysis}

Because the plant samples were mostly polyploid and highly heterozygous, the EST-SSR and PnSSR markers obtained for both Paspalum accessions and $\mathrm{F}_{1}$ hybrids were treated as discrete characters and scored as present (1) or absent (0). Genetic similarity between each pair of accessions was calculated using the Dice (1945) index. Clustering analysis was performed by unweighted pair group method with arithmetic mean (UPGMA) (Sneath and Sokal, 1973) using the NTSYSpc software package (version 2.02; Rohlf, 1997). An estimate of the confidence limits for the grouping produced by the dendrogram was obtained by performing 5000 bootstrap resampling with WinBoot (Yap and Nelson, 1996).

\section{Mapping Procedure}

Expressed sequence tag-SSR and genomic SSR markers were integrated into the $P$. notatum genetic linkage map developed by Stein et al. (2007). Different allelic configurations were expected in the progeny (Ritter et al., 1990). Markers were classified according to their segregation type as maternal, paternal, or biparental when they originated in Q4188, Q4117, or in both genotypes, respectively. For mapping, maternal and paternal markers segregating in a 1:1 (presence:absence) ratio and biparental markers segregating in a 3:1 ratio were used. In addition, a group of paternal markers showing distorted segregation ratios (1:3-1:6) were included because apospory (a component of apomictic reproduction) showed this type of segregation distortion in the species (Martínez et al., 2003; Acuña et al., 2009).

Two segregation datasets were constructed for each parental genotype. Linkage analyses were performed using the mapping software MapMaker/EXP 3.0 (Lander et al., 1987) and JoinMap 3.0 (Van Ooijen and Voorrips, 2001). Initially, new markers scored in the population were integrated into the existing genetic map using MapMaker 3.0 and the command "assign" (logarithm of the odds [LOD] $>2$ as default). The best position of each marker in its linkage group was determined with the command "try." Markers not assigned to an existing linkage group (together with a set of 45 ungrouped AFLP markers from previous work) were grouped using the "group" command (LOD 6-3). The order of markers in each new linkage group was determined using the commands "compare" or "order." Biparental (3:1) markers were incorporated using JoinMap 3.0; their genotypic data were added to each parental data file and linkage analysis was performed with the "fixed order" option (LOD 10-3). Map distances were calculated with Kosambi's mapping function (Kosambi, 1944). Markers were named with their original designation (e.g., cn137, ksum3, or
PN03-A51) followed by a letter corresponding to its maternal $(f)$ or paternal $(m)$ origin and a second letter indicating its relative migration in the gel. The letter $B$ was added before the name of biparental markers. Paspalum notatum linkage groups were named as described by Stein et al. (2007).

\section{Sequence Comparison and In Silico Mapping}

Reference sequences of wEST-SSR and PnSSR markers were retrieved from the National Center for Biotechnology Information database in November 2011 (http://www.ncbi.nlm.nih. gov/ [accessed 10 Oct. 2011]). Fragments of PnEST-SSR markers experimentally mapped in $P$. notatum were cut from the gels, eluted in $500 \mathrm{mM} \mathrm{NH}_{4} \mathrm{C}_{2} \mathrm{H}_{3} \mathrm{O}_{2}$ (ammonium acetate) and 1 $\mathrm{mM}$ EDTA at $\mathrm{pH} 8.0$, and re-amplified using the corresponding primers. The amplification products were cloned in $\mathrm{pGEM}-$ T Easy Vectors (Promega) and sequenced in both directions by Macrogen Inc. (Seoul, Korea). Each PnEST-SSR was examined for the presence of the PCR primers using Sequencher 4.10.1 (Gene Codes, 2011). Moreover, the presence of the SSR motif in the original EST was manually analyzed. Sequences were aligned using ClustalW on the European Molecular Biology Laboratory-European Bioinformatics Institute website (http:// www.ebi.ac.uk/Tools/msa/clustalw2/ [accessed 31 Jan. 2013]).

In silico mapping experiments were performed to determine the putative locations of wEST-SSR sequences in maize as well as the putative positions of $P n S S R$ sequences in both rice and maize genomes. Analyses were performed using the basic local alignment search tool for nucleotide sequences (BLASTn) (Altschul et al., 1990) tool of the GRAMENE (http://www. gramene.org [accessed 19 Nov. 2011]) and MaizeSequence (http://www.maizesequence.org [accessed 19 Nov. 2011]) databases. Alignments parameters were (i) specific database: working gene set transcripts, (ii) search sensitivity: no optimization, (iii) maximum E-value for reports alignments: 0.01, (iv) filter: none, and (v) repeat masker: not selected. Sequences with at least $60 \%$ identity over at least $60 \%$ of the query sequence length at E-value $<0.01$ were considered putative orthologs (Podio et al., 2012). Distances (in cM) between markers in the P. notatum and rice maps were derived from the linkage analysis described above and from $\mathrm{Yu}$ et al. (2004b), respectively. Distances (in cM) between markers mapped in silico on the maize map were estimated by converting physical distance in base pairs to centimorgans according to Ahn and Tanksley (1993).

\section{RESULTS}

\section{Transferability and Polymorphism of Wheat Expressed Sequence Tag-Simple Sequence Repeat and Paspalum notatum Simple Sequence Repeat Markers to Paspalum Species}

A set of 55 primer pairs for wEST-SSR markers (detecting 128 loci evenly distributed on the rice map) was first analyzed in the P. notatum tetraploid genotypes Q4188 and Q4117. These plants were selected for the screening step because they were the parents of an $\mathrm{F}_{1}$ progeny used for map construction and localization of the ACL in the species (Stein et al., 2007). Of the 55 primer pairs tested, 40 (72.72\%) amplified 
Table 2. Transferability of wheat expressed sequence tag-simple sequence repeats to and cross-species amplification of Paspalum notatum simple sequence repeats in Paspalum species.

\begin{tabular}{|c|c|c|c|c|c|c|c|c|c|c|c|c|c|c|c|c|c|c|c|c|}
\hline $\begin{array}{l}\text { Paspalum } \\
\text { species }\end{array}$ & $\begin{array}{l}\text { Group or } \\
\text { subgenus }\end{array}$ & $\frac{\overparen{D}}{\mathrm{C}}$ & $\frac{\bar{\infty}}{\frac{1}{0}}$ & $\frac{O}{\frac{D}{c}}$ & $\frac{\stackrel{\mathbb{N}}{\frac{T}{C}}}{\frac{1}{\delta}}$ & $\frac{\bar{\tau}}{\bar{\tau}}$ & $\frac{\stackrel{N}{ \pm}}{\frac{\Sigma}{\delta}}$ & $\frac{\frac{m}{I}}{\frac{\mathscr{T}}{\mathfrak{L}}}$ & $\frac{\stackrel{10}{ \pm}}{\frac{5}{c}}$ & 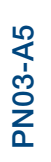 & 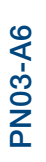 & 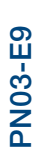 & 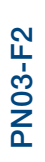 & 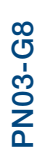 & 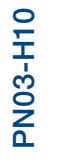 & $\begin{array}{l}\text { N } \\
\frac{1}{1} \\
\text { Oे } \\
\text { Z } \\
\text { L }\end{array}$ & 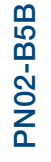 & 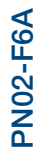 & 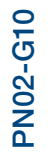 & 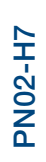 \\
\hline P. lenticulare & Plicatula & $--^{\dagger}$ & $+^{\ddagger}$ & + & + & + & + & + & + & + & - & - & + & + & - & + & - & + & + & + \\
\hline P. guenoarum & & - & + & + & + & + & + & + & + & + & - & - & + & + & - & + & + & + & + & + \\
\hline$P$. atratum & & - & + & + & + & + & + & + & + & + & - & - & + & - & - & + & + & + & + & - \\
\hline P. nicorae & & - & + & + & + & + & + & + & - & + & - & - & + & - & - & - & - & + & + & - \\
\hline P. buckleyanum & Livida & - & + & + & + & + & + & + & + & + & - & - & + & - & - & + & + & + & + & + \\
\hline P. urvillei & Dilatata & - & + & + & + & + & + & + & - & + & - & - & - & - & - & + & + & - & + & + \\
\hline P. dilatatum & & - & + & + & + & + & + & + & + & + & - & - & + & - & - & + & + & + & + & - \\
\hline P. unispicatum & Decumbentes & - & + & + & + & + & + & + & + & + & - & - & - & - & - & + & + & + & - & - \\
\hline P. notatum & Notata & + & + & + & + & + & + & + & + & + & + & + & + & + & + & + & + & + & + & + \\
\hline P. simplex & Anachyris & - & - & + & - & + & + & + & + & + & - & - & + & - & - & - & + & - & - & - \\
\hline
\end{tabular}

$\dagger_{-}$, no amplification products detected.

${ }_{+}+$, at least one band of the expected size was amplified in one accession of the species.

bands of the approximately expected size (between 100 and $500 \mathrm{bp}$ ) and showed polymorphism between the genotypes. Optimal annealing temperatures ranged from 58 to $65^{\circ} \mathrm{C}$ and between 1 and 15 bands were amplified in the two genotypes. On average, wEST-SSRs generated 6.62 bands per primer pair per genotype with at least one polymorphic fragment between Q4188 and Q4117. The remaining $15(27.27 \%)$ primer pairs either did not amplify or showed unspecific patterns and were not further analyzed. A similar assay using the $11 \mathrm{PnSSR}$ primer pairs reported by Cidade et al. (2009) showed, as expected, that all primer combinations amplified two to four fragments in both Q4188 and Q4117 and revealed clear polymorphisms between genotypes.

After this initial screening, a subset of eight wESTSSR primer pairs (cnl64, cnl81, cnl119, cnl124, cnl141, cnl142, cnl143, and cnl145) with well-defined banding patterns and the $11 \mathrm{PnSSR}$ primer combinations were used to analyze 32 Paspalum accessions (Table 1). Transferability of wEST-SSR was considered positive when an amplification product was detected in at least one accession of a given species. The eight selected primer pairs produced PCR fragments within the expected size range in at least one accession (Table 2). Four primer pairs (cnl119, cnl141, cnl142, and cnl143) amplified markers in all 10 species tested, three (cnl81, cnl124, and cnl145) amplified in eight to nine species, and one (cnl64) amplified only in P. notatum. The overall transferability ratio of these wEST-SSR primer pairs to Paspalum spp. was $83.75 \%$. A total of 156 EST-SSR bands were obtained among the 32 accessions, with an average of 19.5 bands per primer pair. The number of markers obtained per primer combination was highly variable. Primer pair cnl142 produced the most bands (62) followed by cnl141 (36), but cnl145 and cnl64 amplified only six and three fragments, respectively. Likewise, there were large differences among accessions. Triploid P. notatum (Q4295) and hexaploid Paspalum buckleyanum Vasey
(Q4303) had the most amplified bands (totals of 43 and 40, respectively) while tetraploid $P$. urvillei (Q4226) produced the fewest (14). In general, accessions of the informal groups Notata and Plicatula showed the highest average number of markers per primer pair (8.10 and 5.1, respectively), and accessions of the Decumbentes group showed the lowest (3.0). The average presence:absence frequency of EST-SSR markers across all accessions was 0.200 , with a minimum and maximum of 0.03 to 0.69 , respectively. Evaluation of genomic $P n S S R s$ indicated that all primer pairs amplified well in accessions of $P$. notatum but were much less effective in the other species analyzed (Table 2). PN03-A5 amplified markers in all 10 species (average 2.3 bands per accession), but PN03-A6, PN03-E9, and PN03-H10 amplified only in P. notatum, with averages of 6.0, 3.7, and 6.0 bands, respectively. The overall rate of cross-species amplification of PnSSRs was $55.45 \%$. A total of 104 bands were obtained across all accessions, with an average of 9.45 informative markers per primer pair. Paspalum notatum accessions had, on average, 4.46 amplified bands per primer pair followed by P. buckleyanum (1.36) and P. guenoarum (1.15). The lowest average number of bands was detected in the Decumbentes group (0.59). The average presence:absence frequency of PnSSR markers was 0.205 , with a minimum and maximum of 0.03 to 0.84 , respectively.

\section{Clustering Analysis}

The applicability of wEST-SSR markers for phylogenetic analysis in the genus was evaluated by conducting a clustering analysis of presence:absence data of the 156 EST-SSR bands. The PnSSRs were not analyzed because they worked mostly on $P$. notatum individuals and few markers were obtained in the other accessions. Analysis of wEST-SSR data showed that Dice's similarity coefficients between species ranged from 0.13 (accessions Paspalum lenticulare Kunth V11725 and Paspalum simplex Morong 


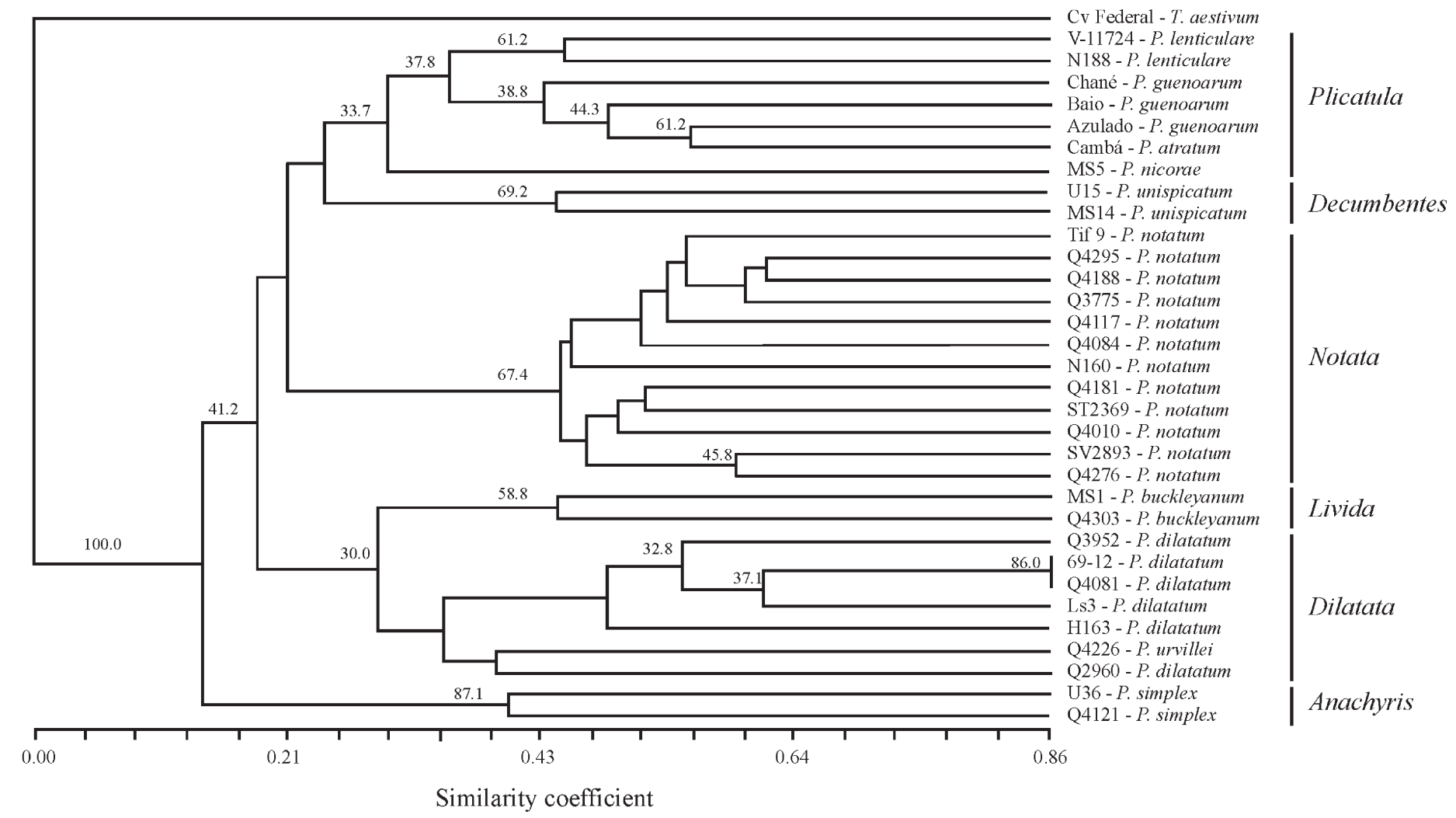

Figure 1. Dendrogram of wheat expressed sequence tag-simple sequence repeats based on Dice similarity index of 32 Paspalum accessions having 156 polymorphic bands. Triticum aestivum cultivar Federal was used as outgroup. Species are indicated on the right. Vertical bars indicate informal taxonomic group or subgenus. The bootstrap values (percentage out of 5000 replicates) are given for the nodes with greater than $30 \%$ support.

Q4125) to 0.56 (accessions P. guenoarum Azulado and P. atratum Cambá), confirming a high level of genetic variation in the material studied. The maximum similarity coefficient (0.86) was detected between two accessions of P. dilatatum (69-12 and Q4081) (Fig. 1).

Clustering analysis grouped accessions by species and taxonomic group (Fig. 1). The dendrogram could be divided at about 30\% similarity into four clusters. Cluster I contained two subclusters, one including species of the Plicatula group (P. lenticulare, Paspalum limbatum Henrard, P. guenoarum, P. atratum, and Paspalum nicorae Parodi) (bootstrap $P$-value for this group was 33.7\%) and the other a species of the Decumbentes group [Paspalum unispicatum (Scribn. \& Merr.) Nash] (bootstrap P-value 69.2\%). Cluster 2 included all $P$. notatum individuals, which grouped together irrespective of ploidy level and mode of reproduction (bootstrap P-value for this group was 67.4\%). Cluster 3 comprised two subclusters, one including the two accessions of $P$. buckleyanum (Livida group) (bootstrap $P$-value $=58.8 \%$ ) and the other with the six individuals of $P$. dilatatum plus the single accession of P. urvillei although no significant bootstrap support was found for this grouping. Cluster 4 contained only accessions of $P$. simplex from the subgenus Anachyris (bootstrap P-value $=87.1 \%$ ).

\section{Mapping Wheat Expressed Sequence Tag- Simple Sequence Repeat and Paspalum notatum Simple Sequence Repeat Markers in Tetraploid Paspalum notatum}

Thirty-one wEST-SSR primer combinations showing polymorphisms between the tetraploid genotypes Q4188 and Q4117 were used for mapping experiments. Of these, 28 produced well-defined segregating markers from one or both genotypes (Fig. 2a) while the remaining three showed unspecific patterns and were not further analyzed. A total of 216 EST-SSR segregating markers were obtained in the population (Supplemental Table S3). Segregation analysis showed that 106 maternal and paternal (uniparental) markers (49.07\%) segregated in a 1:1 ratio and must correspond to single-dose alleles. Another group of 17 uniparental markers $(7.87 \%)$ segregated in $3: 1$ or $5: 1$ ratio consistent with double-dose alleles, and a third group of 41 uniparental markers (18.98\%) showed distorted segregation ratios that did not fit in any expected value for an autotetraploid species. Biparental markers showed 25 (11.57\%) fragments segregating as single-dose alleles (3:1 ratio) while 27 (12.50\%) exhibited distorted segregation ratios (Table 3). Analyses using genomic PnSSRs showed that all primer pairs produced segregating fragments in the progeny (Fig. 2b) (Supplemental Table S3). A total of $39 \mathrm{PnSSR}$ markers were obtained in the population. 
$\begin{array}{llll}\text { MW } 1 & 2 & \text { F1 progeny }\end{array}$

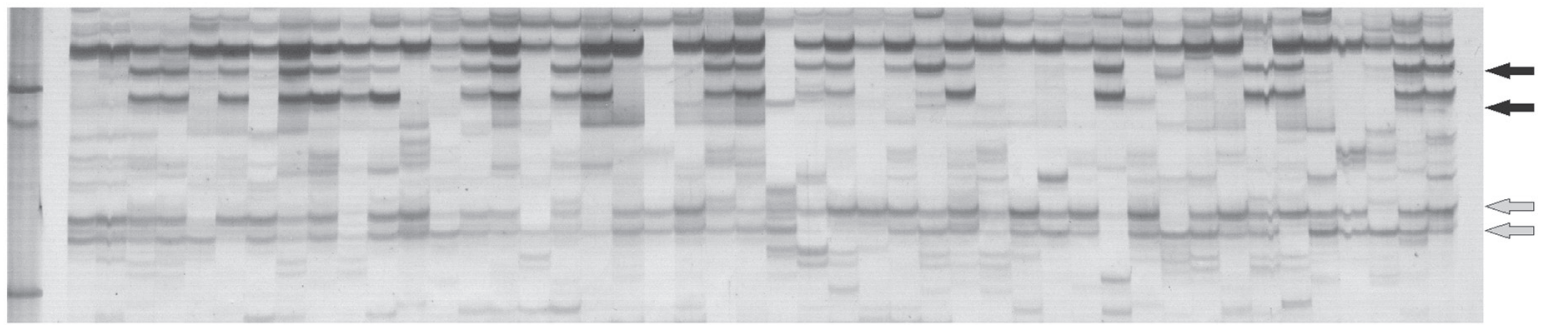

b $\quad$ MW $\quad 1 \quad 2$

F1 progeny

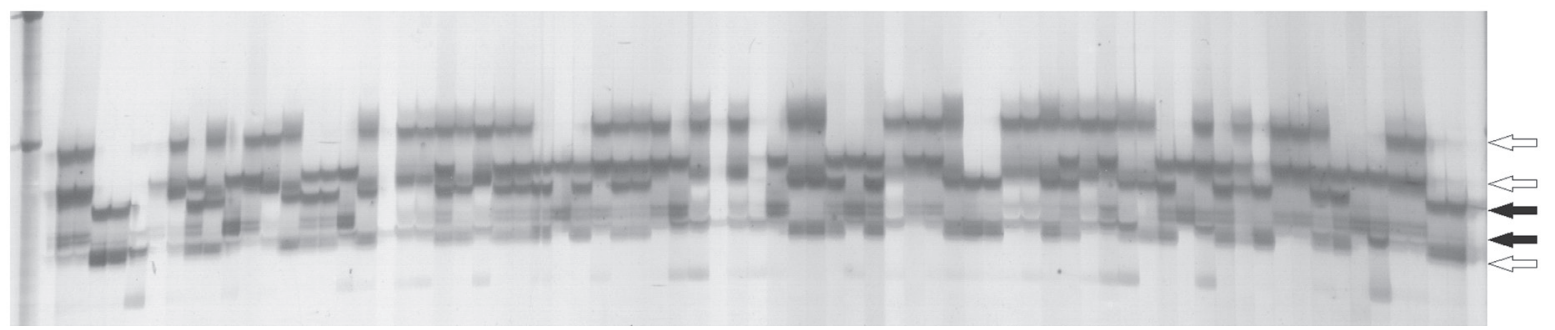

Figure 2. Mapping analysis of wheat expressed sequence tag (EST)-simple sequence repeats (SSRs) and Paspalum notatum genomic SSR (PnSSR) markers on P. notatum. Silver-stained acrylamide gels showing the amplification pattern of wheat EST-SSR cnl142 (A) and PnSSR PN03-H10 (B) markers. 1 and 2: parental genotypes Q4188 and Q4117 loaded in duplicate. Black, white, and grey arrows indicate paternal, maternal, and biparental markers scored in the progeny, respectively. MW, 100-bp molecular weight standard (Promega).

Table 3. Number of wheat expressed sequence tag (EST)-simple sequence repeats (SSRs) and Paspalum notatum genomic simple sequence repeat (PnSSR) markers scored in each category of segregation ratio in tetraploid Paspalum notatum.

\begin{tabular}{|c|c|c|c|c|c|c|c|}
\hline \multirow{3}{*}{$\begin{array}{l}\text { Marker } \\
\text { type }\end{array}$} & \multirow[b]{3}{*}{ Class } & \multicolumn{5}{|c|}{$\begin{array}{l}\text { Allelic configuration and expected } \\
\text { segregation ratio }\end{array}$} & \multirow[b]{3}{*}{ Total } \\
\hline & & \multirow{2}{*}{$\frac{\mathrm{SDA}^{\dagger}}{1: 1}$} & \multicolumn{2}{|c|}{$\mathrm{DDA}^{\ddagger}$} & \multicolumn{2}{|c|}{ Dist.§ } & \\
\hline & & & $3: 1$ & $5: 1$ & $1: 3-1: 6^{*}$ & Other** & \\
\hline \multirow[t]{3}{*}{ EST-SSR } & Maternal & 54 & 7 & 2 & 15 & 8 & 86 \\
\hline & Paternal & 52 & 7 & 1 & 11 & 7 & 78 \\
\hline & Biparental & - & 25 & - & - & 27 & 52 \\
\hline \multirow[t]{3}{*}{ PnSSR } & Maternal & 13 & 2 & 2 & 0 & 1 & 18 \\
\hline & Paternal & 14 & 0 & 2 & 1 & 0 & 17 \\
\hline & Biparental & - & 4 & - & 0 & 0 & 4 \\
\hline
\end{tabular}

*Significant at the 0.05 probability level.

**Significant at the 0.01 probability level.

tSDA, single-dose alleles.

¥DDA, double-dose alleles.

\$Dist., markers with distorted segregation ratios.

Twenty-seven (69.23\%) corresponded to maternal or paternal markers segregating as single-dose alleles, six $(15.38 \%)$ segregated in $3: 1$ or $5: 1$ ratios (as double-dose alleles), two $(5.12 \%)$ showed distorted segregation ratios, and four (10.25\%) biparental PnSSR markers segregated in 3:1 ratio (Table 3).

For mapping analysis, 67 new loci segregating in a 1:1 ratio from Q4188 (54 EST-SSRs and 13 genomic SSRs), 66 from Q4117 (52 EST-SSRs and 14 genomic SSRs), and 29 (25 EST-SSRs and four PnSSRs) biparental markers were considered. In addition, 12 paternal markers (11
EST-SSRs and one PnSSR) showing distorted segregation ratios $1: 3$ to $1: 6$ were included in the analysis. The mapping procedure allowed the incorporation of 38 (29 EST-SSRs and nine SSRs) and 64 (48 EST-SSRs and 16 SSRs) new markers into the existing female (Q4188) and male (Q4117) linkage maps, respectively. Both types of markers were scattered through the genome, and no close linkages were found between any of them; markers mapped to 19 of 34 and 33 of 45 linkage groups of Q4188 and Q4117, respectively. On average, 1.11 (range 0-7) and 1.42 (range 0-6) markers per linkage group were localized in the respective genomes. The addition of these markers increased by six and eight the number of known linkage groups and extended the genetic distances covered by the maps from 1815 to $1920 \mathrm{cM}(5.72 \%)$ and from 2708 to 3118 cM (15.14\%) in Q4188 and Q4117, respectively.

In particular, these markers (ksum206pbd, ksum219pbd, and PN03-F2) mapped in coupling phase to the linkage group M17a at both sides of the ACL (Fig. 3). The two wEST-SSRs (Ksum206 and Ksum219) from which they derived mapped to a region of rice chromosome 12 previously associated with the trait (Martínez et al., 2003; Stein et al., 2004; Pupilli et al., 2004). Both markers also flanked the RFLP marker C1069, which is completely linked to apospory in several Paspalum species (Pupilli et al., 2004; Hojsgaard et al., 2011). This group of three markers had the same order and similar distances in rice and $P$. notatum maps, confirming the high degree of synteny between the two species in this specific genomic region reported by Pupilli et al. (2004). Other wEST-SSR markers on rice 


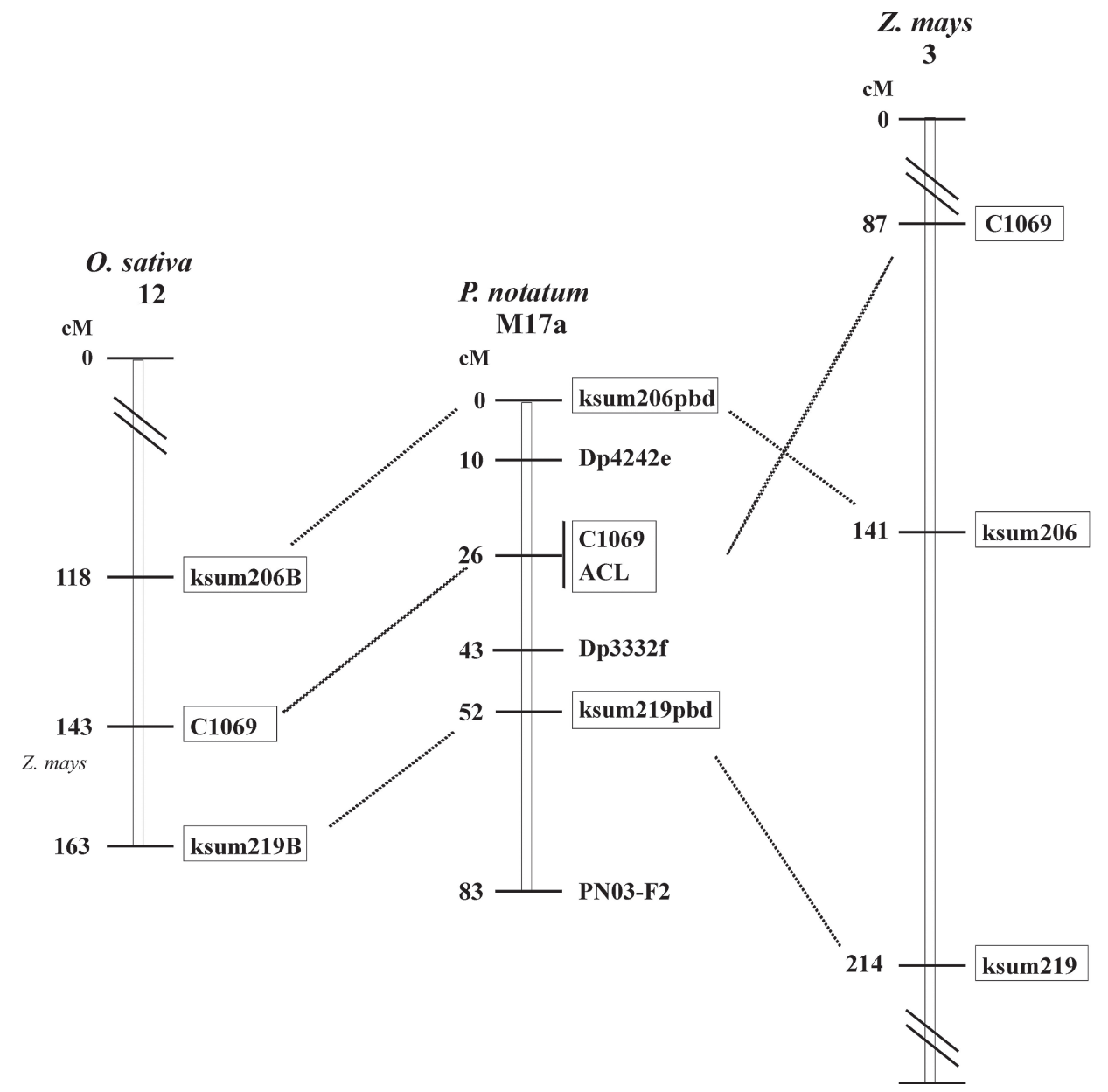

Figure 3. Genetic map of Paspalum notatum linkage group M17a carrying the apospory locus. Markers ksum206pbd, ksum219pbd, and PN03-F2 mapped in coupling phase at both sides of the apospory controlling locus (ACL) of the species. Both wheat expressed sequence tag-simple sequence repeats also flank the restriction fragment length polymorphism marker C1069, which is completely linked to apospory in several Paspalum species. The positions of the same markers on rice chromosome 12 and the putative orthologous sequences mapped in silico on maize chromosome 3 are shown to the left and right, respectively.

chromosomes 12 and 2 mapped to the putative linkage group homologous to M17a (Table 4). Moreover, in silico mapping analysis (see below) detected putative orthologous sequences of wEST-SSR markers ksum206 and ksum219 and the RFLP C1069 on maize chromosome 3.

\section{Analysis of Paspalum notatum Expressed Sequence Tag-Simple Sequence Repeat Sequences}

To examine experimentally mapped EST-SSR markers in $P$. notatum at the DNA sequence level, 32 amplicons (150-450 bp) of $13 \mathrm{wEST}-S S R$ primer pairs were analyzed by the presence of the primer sequences, the microsatellite repeat, and the similarity with the original wheat EST fragment. All fragments contained the corresponding primers and most $(93.75 \%)$ perfectly matched the oligonucleotide sequence. Thus, all fragments were validated as $P_{n} \mathrm{EST}-$ SSR markers and unspecific amplifications were discarded. In addition, all amplicons contained the expected microsatellite motif at least once, but only a few had perfect repetitions of two or three motifs. The majority of sequences contained 1 to 10 imperfect SSRs (Supplemental Table S4). Alignments with the corresponding wheat EST showed identity values ranging from 46.23 to $55.27 \%$ (Supplemental Table S4), indicating that although primer sites were well conserved, internal sequences varied. Nevertheless, in three cases, $P_{n} \mathrm{EST}-\mathrm{SSR}$ sequences (e.g., Pnksum10, Pnksum141, and Pnksum205) matched the corresponding wEST in a general BLASTn search on the wheat genome database (http://blast.jcvi.org [accessed 31 Jan. 2013]). On the other hand, alignments of PnEST-SSRs derived from the same primer pair showed variable identity values. Fragments amplified by primer pairs cnl132 and ksum230 had 76.40 and $67.47 \%$ identity, respectively, and other primer pairs yielded fragments with values ranging from 43.35 to $56.10 \%$, which may correspond to different sequences (Supplemental Table S4).

\section{In Silico Mapping and Comparative Analysis}

The sequences of $28 \mathrm{wEST}-\mathrm{SSR}$ and $11 \mathrm{PnSSR}$ markers experimentally mapped in $P$. notatum were mapped in silico (BLASTn) on the MaizeSequence (http:// 
Table 4. Experimental and in silico mapping of wheat expressed sequence tag-simple sequence repeats and Paspalum notatum simple sequence repeat markers in $P$. notatum, rice, and maize genomes.

\begin{tabular}{|c|c|c|c|c|}
\hline Primer pair & P. notatum accession $\mathrm{Q} 4188^{\dagger}$ & P. notatum accession $\mathrm{Q} 4117^{\dagger}$ & Rice chromosome ${ }^{\ddagger}$ & Maize chromosome ${ }^{\ddagger}$ \\
\hline cnl64 & - & M1a & 2 & 2,4 , and 5 \\
\hline cnl81 & F6a, F9a, F21, and F24 & M4a, M4b, M6a, and M27 & 12 & 3 and 4 \\
\hline cnl119 & - & M9b and M20a & 4 & 6 \\
\hline cnl126 & $\mathrm{F} 14$ & $\mathrm{M} 2 \mathrm{C}$ and $\mathrm{M} 14 \mathrm{a}$ & 4 & 3 and 8 \\
\hline cnl132 & F2, F3b, and F26 & $\mathrm{M} 2 \mathrm{a}$ & 9 & 1,3 , and 7 \\
\hline cnl141 & $\mathrm{F} 1 \mathrm{~b}, \mathrm{~F} 2, \mathrm{~F} 6 \mathrm{a}$, and F11a & M1a and M18a & 5 & 4 and 10 \\
\hline cnl142 & - & M1a, M9a, M9b, M16a, and M23a & 3,4 , and 11 & $1,2,3,4,5$, and 10 \\
\hline cnl143 & - & M8a and M23a & 7 & 2,3 , and 10 \\
\hline cnl144 & $n d^{\S}$ & nd & 8 & 3,4 , and 10 \\
\hline cnl145 & - & M6b and M9c & 9 and 11 & $1,2,5,6$, and 8 \\
\hline cnl157 & $\mathrm{F} 13$ & - & 7 & $4,5,6$, and 10 \\
\hline cnl158 & - & M17b? & 2,7 , and 8 & $3,4,5,7$, and 8 \\
\hline ksum3 & $\mathrm{F} 2$ & M10b" & 1,4, and 12 & - \\
\hline ksum10 & $\mathrm{F} 10$ & M6b and M30 & $2,4,5$, and 10 & $2,5,6$, and 8 \\
\hline ksum24 & $\mathrm{F} 5 \mathrm{a}$ & $\mathrm{M} 1 \mathrm{~b}, \mathrm{M} 22 \mathrm{a}, \mathrm{M} 23 \mathrm{a}$, and M30 & $2,4,6$, and 7 & $2,4,5,6,7,9$, and 10 \\
\hline ksum27 & nd & nd & 3 & $1,2,7,9$, and 10 \\
\hline ksum28 & nd & nd & 4,7 , and 10 & 1,3 , and 7 \\
\hline ksum67 & nd & nd & 2 and 4 & $1,2,4,5$, and 8 \\
\hline ksum108 & $\mathrm{F} 1 \mathrm{~b}$ and $\mathrm{F} 5 \mathrm{a}$ & M9a and M17b" & $4,8,11$, and 12 & 2,7, and 10 \\
\hline ksum140 & - & M3b & $1,2,7$, and 11 & 7 \\
\hline ksum141 & F9a and F11a & - & $5,7,10$, and 11 & 1 and 9 \\
\hline ksum153 & F24 & M6a & 8 and 11 & 4 \\
\hline ksum186 & - & M6b & 6 and 7 & 2, 3, 4, and 5 \\
\hline ksum202 & $\mathrm{F} 3 \mathrm{c}$ and $\mathrm{F} 11 \mathrm{a}^{\mathrm{l}}$ & $\mathrm{M} 3 \mathrm{c}, \mathrm{M} 8 \mathrm{a}$, and $\mathrm{M} 14 \mathrm{a}$ & 1,2 , and 7 & $1,2,3,4,6,7$, and 8 \\
\hline ksum205 & F3a, F5a, F6a, and F11a & M8a, M18b, and M29 & 3,6 , and 8 & $2,5,8,9$, and 10 \\
\hline ksum206 & - & $\mathrm{M} 17 \mathrm{a}$ & 1,7 , and 12 & $1,3,7$, and 8 \\
\hline ksum219 & - & $\mathrm{M} 17 \mathrm{a}$ & 6,9, and 12 & $1,3,5$, and 8 \\
\hline ksum230 & - & M10b" & $3,4,11$, and 12 & $1,2,3,5,8$, and 10 \\
\hline PN03-A5 & - & $\mathrm{M} 18 \mathrm{a}$ & 1 and 4 & 1 \\
\hline PN03-A6 & F26 & $\mathrm{M} 2 \mathrm{a}, \mathrm{M} 4 \mathrm{a}$, and M25 & - & - \\
\hline PNO3-E9 & - & M10a" & - & - \\
\hline PN03-F2 & $\mathrm{F} 5 \mathrm{c}$ & $\mathrm{M} 17 \mathrm{a}$ and $\mathrm{M} 19 \mathrm{a}$ & - & - \\
\hline PN03-G8 & - & M28 & - & - \\
\hline PN03-H10 & F18 & M20 and M3b & $2,3,4,5,6,9$, and 12 & $2,3,4,5,6,7$, and 8 \\
\hline PN02-A12 & $\mathrm{F} 5 \mathrm{a}$ & - & 9 and 10 & - \\
\hline PN02-B5B & F5a and F23a & - & - & - \\
\hline PN02-F6A & - & M21a & $1,2,3,4,5,6,8,9$, and 11 & $1,2,3,4,5,7,8,9$, and 10 \\
\hline PN02G10 & $\mathrm{F} 5 \mathrm{c}$ & M5a and M11a & - & - \\
\hline $\mathrm{PNO2-H7}$ & - & M6b and M17b & 7 & - \\
\hline
\end{tabular}

†Linkage groups reported by Stein et al. (2007).

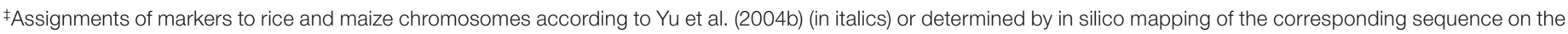

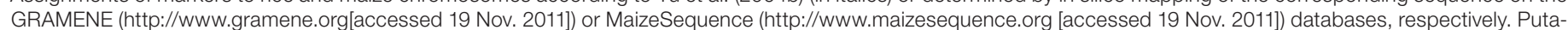

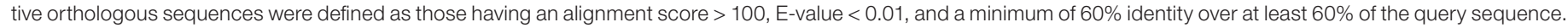
§nd, not determined.

"Putative homologs of the linkage groups carrying the apospory locus according to Stein et al. (2007).

www.maizesequence.org [accessed 19 Nov. 2011]) and GRAMENE (http://www.gramene.org [accessed 19 Nov. 2011]) databases. The BLASTn analysis of wEST-SSR sequences detected 106 putative orthologous sequences distributed on the 10 maize chromosomes (Table 4). As expected, several putative orthologs (average 3.78) were identified for each wEST-SSR sequence. The maximum identity value between wEST-SSR s and maize ESTs was 86.51\% (Supplemental Table S5). In general, alignments covered almost the complete length of the query sequence (score value $>100)$ and in several cases $(38.67 \%)$ the subject sequence included at least one of the oligonucleotides used for marker amplification with a minimum of $65 \%$ identity. Moreover, most subject sequences contained the SSR motif present in the original EST (Supplemental Table S5). Similar analysis performed with $P n S S R$ sequences detected 17 and 21 putative orthologous sequences in the maize and rice genomes, respectively (Table 4) (Supplemental Table 
S6), with identity values ranging from 61.03 to $76.32 \%$ and 61.05 to $71.09 \%$ in maize and rice, respectively.

The relative positions of $28 \mathrm{wEST}-\mathrm{SSR}$ and $11 \mathrm{PnSSR}$ markers mapped in $P$. notatum were compared with their locations in the rice and maize maps. The whole analysis included 102 markers (77 wEST-SSRs and 25 PnSSRs) mapped in $P$. notatum, $66 \mathrm{wEST}-\mathrm{SSR}$ s mapped in rice (Yu et al., 2004b), 106 putative wEST-SSR orthologs in maize, and 17 and 21 putative PnSSR orthologs in maize and rice, respectively (Table 4). In general, EST-SSR that generated more than one marker in $P$. notatum and rice showed more than one positive alignment in the maize genome. For instance, ksum24 detected five loci in $P$. notatum, four loci in rice, and seven alignments in maize. Similar results were obtained with ksum10, ksum108, ksum202, and ksum205. In other cases, only a few loci or putative orthologs were detected in the three species (e.g., cnl64, cnl119, ksum153). Analysis of PnSSR s showed that all primer pairs generated between one and three markers in P. notatum, but only five sequences aligned positively at least once in the rice and maize genomes: three (PN03-A5, PN03-H10, and PN02-F6A) aligned in both species while the other two (PN02-A12 and PN02-FH7) detected sequences only in the rice genome. In addition sequences of PN03-H10 and PN02-F6A had multiple alignments in both species. Comparisons of relative positions of the markers and sequences in the three species showed some similarities. Primer pairs cnl143, ksum202, and ksum205 generated linked markers that mapped in a segment of about $60 \mathrm{cM}$ in the $P$. notatum linkage group M8a. Putative orthologs of these markers were found on chromosome 2 of maize (about $30 \mathrm{cM}$ ) although ksum 205 appeared inverted. Moreover, cnl143 and ksum202 generated fragments that mapped linked in rice chromosome 7 (about 14 cM) (Fig. 4a). Similarly, cnl142, ksum24, and cnl143 generated markers mapped in the P. notatum linkage group M23a (34 cM). Two of these primer pairs (cnl142 and ksum24) defined three loci on rice chromosome $4(45.5 \mathrm{cM})$ and putative orthologs of both markers were found at 8 and $2.6 \mathrm{cM}$ on chromosomes 2 and 10 of maize, respectively. Moreover, ksum24 and cnl143 mapped closely linked in maize chromosome 7 (Fig. 4b). In most cases, the lengths of the chromosome segments covered by the markers were similar between species.

\section{DISCUSSION}

\section{Transference of Wheat Expressed Sequence Tag-Simple Sequence Repeat Markers and Cross-Species Amplification of Genomic Paspalum notatum Simple Sequence Repeat Markers}

In the present work, we selected a group of publicly available wEST-SSR markers to determine their transferability to and polymorphisms in Paspalum spp. and to evaluate their utility for genetic and phylogenetic analyses in the genus. The initial screening for informative wEST-SSR markers was performed on two tetraploid genotypes of $P$. notatum that had been previously used as parents of a mapping population. In this analysis, $72.72 \%$ of wESTSSR primer pairs amplified fragments near the expected size in both samples. Typically, multiple bands amplified per primer pair, indicating that different alleles or loci were detected. Subsequently, a group of eight informative wEST-SSR primer pairs was used to analyze 32 Paspalum accessions. This subset of markers showed an overall transferability of $83.75 \%$ and generated a total of 156 bands across all accessions. This relatively high amplification efficiency in species not closely related to wheat may have occurred because the wEST-SSRs were developed based on a clustering approach that included groups of putative ortholog sequences of different grass species (supercluster analysis) (Kantety et al., 2002; Yu et al., 2004a). Therefore, the informative primer pairs identified by screening on $P$. notatum might detect sequences that are well conserved within Paspalum. High transferability was also found with a different set of wEST-SSRs to Secale cereale L. (rye) and Hordeum vulgare L. (barley) by Zhang et al. (2007). Large differences were detected between primer pairs in the number of amplified markers. In particular, cnl142 amplified the most bands across all species. The original wheat EST was annotated as a cytosolic $60 \mathrm{~S}$ ribosomal protein L9 that is expected to have multiple copies in the genome (Barakat et al., 2001). In contrast, cnl64, derived from an EST encoding a phenylalanine ammonia-lyase (PAL) enzyme, amplified the fewest fragments in the Paspalum species. Only four $P A L$ genes were annotated in Arabidopsis thaliana (L.) Heynh. (Cochrane et al., 2004). Therefore, part of the differences in amplification capacity among primer pairs in our material may be due more to the number of genes in the gene family of each EST than to primer specificity or genomic differences among accessions.

Overall, wEST-SSR primer pairs generated multiple bands. This might be due to the detection of different alleles or loci in the amplifications. Consequently, wESTSSRs were scored as dominant markers. However, wESTSSRs detected a high variation in the genetic similarities between accessions, and informative markers occurred in all species. These results confirm that wEST-SSRs markers are adequate to analyze variability in species of Paspalum for which no marker technologies have yet been developed.

Analysis of PnSSRs indicated that they amplified well in P. notatum accessions while only about half of them amplified in other Paspalum species. The general cross-species amplification success (55.45\%) and the average number of markers amplified per primer combination were lower than those for wEST-SSRs. Similar results were 


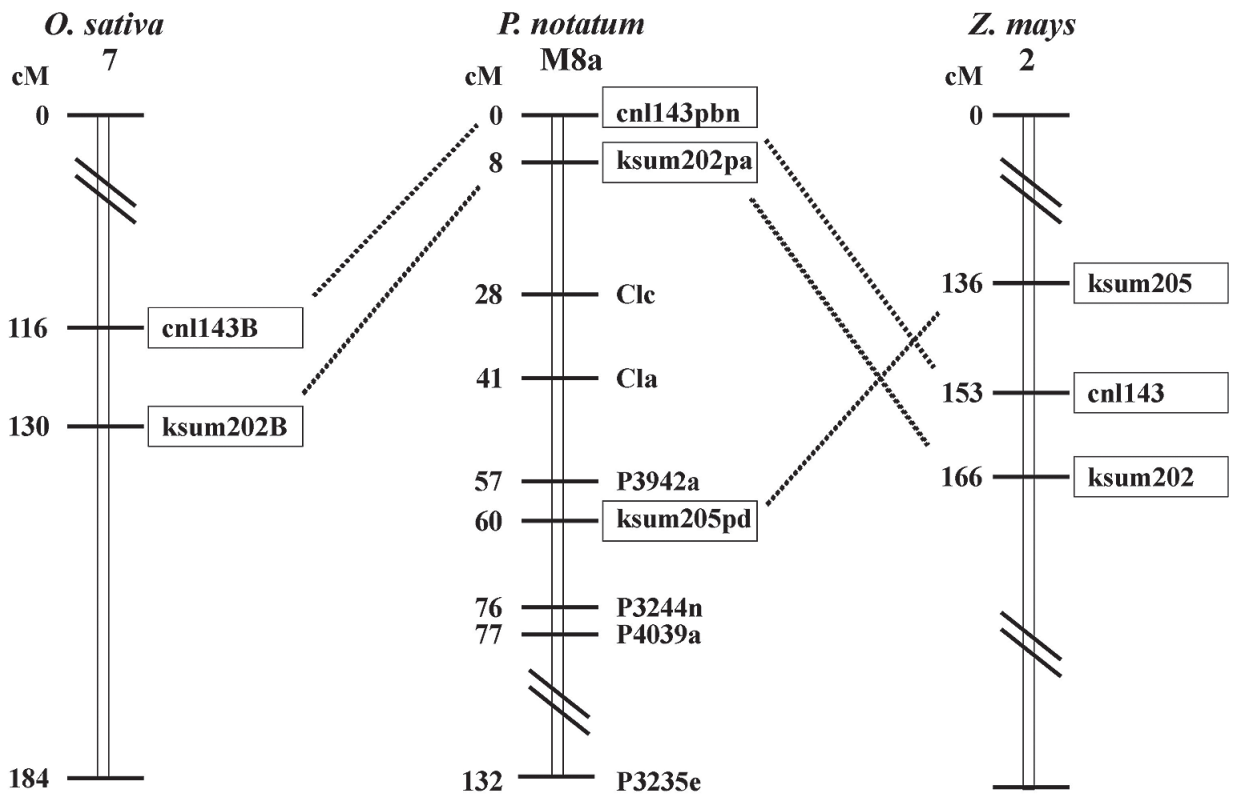

b

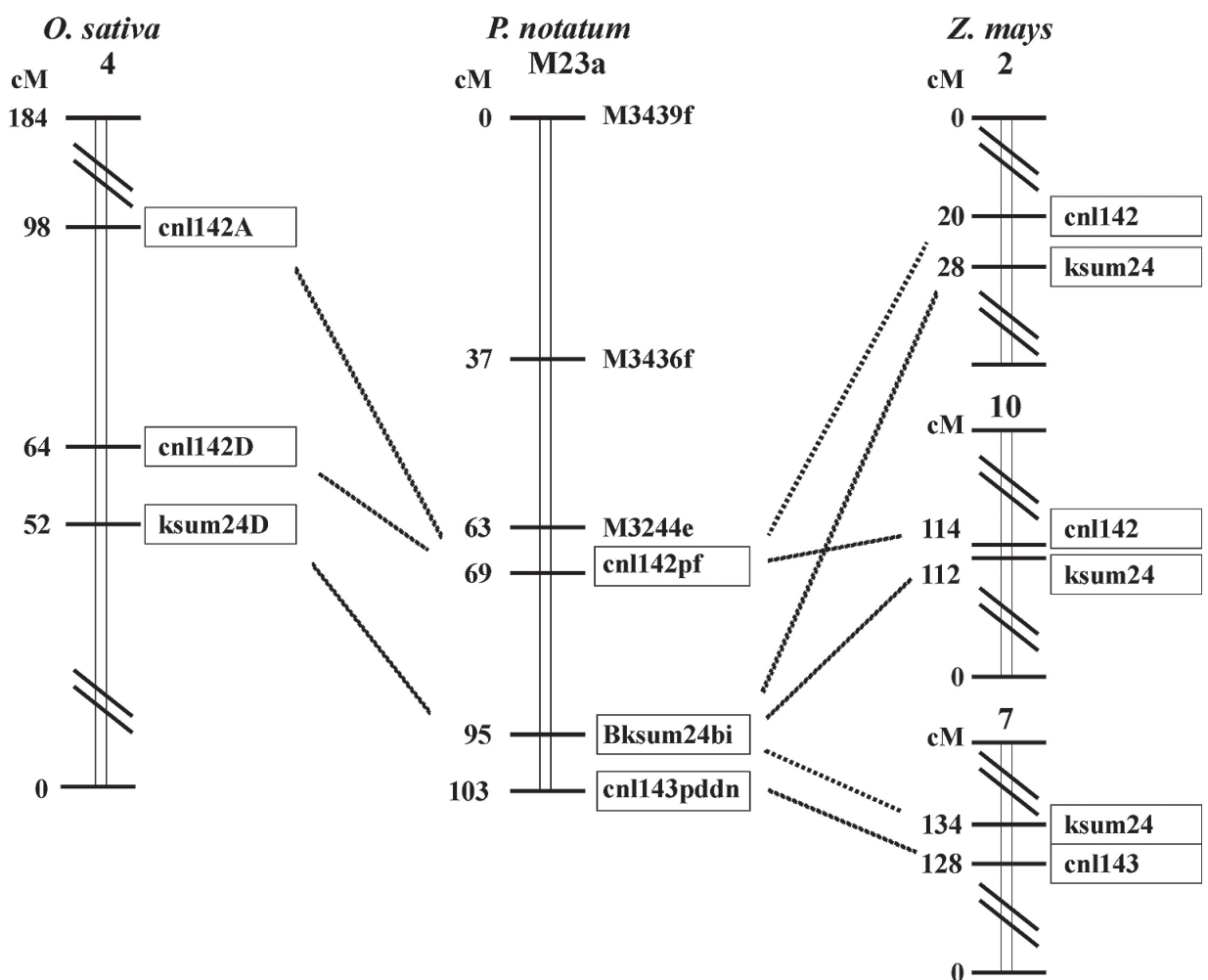

Figure 4. Comparative mapping analysis based on experimentally mapped wheat expressed sequence tag (EST)-simple sequence repeat (SSR) markers on the Paspalum notatum genetic map. A and B: Paspalum notatum linkage groups M8a and M23a and their putative homologs in rice and maize. Positions of wheat EST-SSR markers in rice chromosomes were obtained from Yu et al. (2004b). Putative location of markers in the maize groups were derived from the in silico mapping assays performed in this work.

obtained with the same set of PnSSR markers in other species of the genus (Paspalum subciliatum Chase, Paspalum cromyorhizon Trin. ex Döll, and Paspalum ionanthum Chase) by Cidade et al. (2009). Therefore, PnSSRs have showed a low level of sequence conservation in the other species of the genus. This is in agreement with previous reports indicating that genomic SSRs showed less efficient transferability than EST-SSRs (Varshney et al., 2005). Considering these results and the few primer pairs available so far, PnSSR appeared to have limited utility for studying variability in natural population of other Paspalum species or for large phylogenetic surveys in the genus. 


\section{Clustering Analysis with Expressed \\ Sequence Tag-Simple Sequence Repeat Markers}

In a clustering analysis, the cladogram resolved four clusters, two (clusters I and III) with multiple species and two (clusters II and IV) with a single species each. Although low confidence limits were obtained for many branches by the bootstrap analysis, some of the relationships are in agreement with those found in previous works using morphological, cytological, and molecular tools. Briefly, cluster I included two subclusters: one integrated by species of the Plicatula group and the other by P. unispicatum of the Decumbentes group. These two informal groups were also sister to one another in a phylogenetic tree based on sequence data of the rpl16 and trnL-F genes (Giussani et al., 2009). Moreover, within the Plicatula group, the three P. guenoarum individuals grouped together, but one (accession Azulado) appeared closely related to accession Cambá of $P$. atratum. This association agrees with a previous taxonomic analysis indicating a close relationship between these species (Zuloaga and Morrone, 2005). In addition, accession MS5 of P. nicorae, a species easy to distinguish within the Plicatula group by the morphology of its rhizomes (Zuloaga and Morrone, 2005), appeared well differentiated in the UPGMA analysis. Accessions of P. lenticulare (V11724 and N188) appeared together in cluster I. Accession N188 was previously classified as a $2 x$ sexual P. limbatum by Espinoza et al. (2001). However, because N188 and V11724 have only minor morphological differences, $P$. limbatum was suggested to be the diploid form of P. lenticulare (Espinoza et al., 2001). Later, Oliveira and Valls (2008) synonymized P. limbatum with $P$. lenticulare. Cluster II contained only accessions of $P$. notatum (Notata group) and was differentiated from the other clusters. The range of genetic similarity between accessions is also in agreement with values obtained in a previous study using the same set of PnSSRs on a large collection of Brazilian, Uruguayan, and Argentine accessions by Cidade et al. (2009). Moreover, a highly similar grouping of $P$. notatum accessions was obtained with data derived from the PnSSR markers used in this work (not shown). These results indicate that both types of markers reveal the same genetic characteristics and can be routinely used for this type of analysis in germplasm collections or natural populations of the species. Cluster III included species of the informal groups Livida (P. buckleyanum) and Dilatata (P. dilatatum and P. urvillei). A sister relationship between these two groups was also found in a previous study based on morphology, ITS, and cpDNA sequence data (Souza-Chies et al., 2006). Although cladistic treatments of the Dilatata group are difficult because those species are allopolyploids and include at least one reticulation event in their evolutionary history (Souza-Chies et al., 2006), our analysis found several notable associations. For instance, accessions of dallisgrass ( $P$. dilatatum) 69-12 and Q4081 were highly similar (0.86). Q4081 is a facultative apomict dallisgrass and 69-12 is one of their off-type progenies, which is a yellowed-anther individual that produced exclusively one embryo sac per ovule of the sexual type (Millot, 1977). Moreover, Ls3 appeared related to both accessions although it was collected at a distant location from Q4081. Morphologically, Ls3 represents common dallisgrass, the apomictic pentaploid $(2 n=5 x=50)$ biotype with a genome formula IIJJX (Burson, 1983) while accession Q4081 corresponds to an apomictic hexaploid $(2 n=6 x$ $=60)$, referred to as the Uruguayan biotype, with a genome formula IIJJXX (Burson, 1991a). The close association found between these accessions was supported by a previous cytogenetic report in which Burson (1991b) demonstrated that chromosomes of the arbitrarily designated $\mathrm{X}$ genome from the pentaploid common biotype (IIJJX) were homologous to the members of the $\mathrm{X}$ genome of the hexaploid Uruguayan biotype (IIJJXX). On the other hand, several $P$. dilatatum accessions appeared related to the tetraploid $(2 n=$ $4 x=40$ ) accession Q4226 of P. urvillei (vaseysgrass). Similar associations between these species have been detected in an RFLP-based genetic diversity analysis by Jarret et al. (1998). In particular, accession Q3952 is a sexual yellow-anthered tetraploid $(2 n=4 x=40)$ biotype of dallisgrass called $P$. dilatatum subsp. flavescens Roseng. et al. A close phylogenetic relationship between these two species has been determined indirectly by interspecific crosses concluding that they had the same genomic formula (IIJJ) (Burson and Bennett, 1972; Burson, 1983). Furthermore, in this study we included one sexual tetraploid biotype of $P$. dilatatum (accession Q2960; Virasoro type), also characterized by yellow anthers but distinguished by its larger and multinerved spikelets (Caponio and Quarin, 1987). Crosses between the Virasoro type of P. dilatatum (Q2960) and P. urvillei were more fertile than any other crosses between different biotypes of $P$. dilatatum and $P$. urvillei, indicating a good degree of genome homology (Caponio and Quarin, 1990). Cluster IV was composed only of the two accession of $P$. simplex included in this work and appeared to be more distantly related to the other material analyzed. Indeed, $P$. simplex is taxonomically distinct from the others species analyzed and is included in a different subgenus (Anachyris) (Zuloaga and Morrone, 2005). In this respect, our cladogram is in agreement with a consensus phylogenetic tree of cpDNA sequence data in which one accession of $P$. simplex and other species of subgenus Anachyris (Paspalum malacophyllum Trin. and Paspalum usteri Hack.) formed a subcluster of the group containing accessions of the informal groups Livida, Notata, and Plicatula (Rua et al., 2010). These concordances might be based on these types of nuclear markers tag gene sequences that are expected to provide morphological and physiological features to the individuals. Therefore, EST-SSR markers can help to resolve phylogenetic relationships within the genus, avoiding problems of close morphology, the allopolyploid nature of many of species, and reticulate evolution. 


\section{Mapping Analysis}

Mapping experiments were performed to analyze the distributions of wEST-SSR and PnSSR markers in the $P$. notatum genome and explore their utility for anchoring the existing linkage map of the species. A group of primer pairs detecting loci distributed in the rice map (Yu et al., $2004 \mathrm{~b}$ ) were assayed in a tetraploid mapping population previously used to determine the mode of inheritance of common P. notatum and to localize its ACL (Stein et al., 2007). Because the rice genome is a model for grasses (Devos and Gale, 2000), this strategy intended to cover different regions of the P. notatum genome. Overall, several segregating markers were obtained with most wESTSSR primer pairs. On the other hand, two to six segregating fragments were detected with each PnSSR primer combination. As expected because of the highly heterozygous condition of the parents, different types of segregation ratios were observed. However, most markers segregated as uniparental single-dose alleles and therefore may be used for mapping analysis. A group of markers from the male parent (Q4117) showed distorted segregation ratios similar to those observed for apospory. These results are compatible with the segregation ratios of AFLP markers observed in the same population by Stein et al. (2007).

Genetic mapping of 102 new markers showed that both EST-SSRs and PnSSRs were widely distributed in the $P$. notatum genome and not clustered or concentrated around centromeric regions. This characteristic may facilitate covering the genome and might speed map construction based on anchor markers. Moreover, because most of the wESTSSR markers had functional annotations, mapping this type of marker can also help to localize sequences of agronomic interest. Markers mapped in this work allowed the extension of the current map, increasing the number of linkage groups in both parental genotypes. Interestingly, two EST-SSRs and one PnSSR marker mapped at either side of the ACL. Both wEST-SSR markers mapped on rice chromosome 12 to a region previously associated with apospory (Martínez et al., 2003; Pupilli et al., 2004; Stein et al., 2007). These markers defined a segment of about $45 \mathrm{cM}$ around the ACL with a high degree of synteny with a segment on the long arm of rice chromosome 12 and may include the apospory triggers in the species. In addition, putative orthologs of these markers were found on maize chromosome 3 , in agreement with a previous report by Podio et al. (2012).

\section{Expressed Sequence Tag-Simple Sequence Repeat Sequence Analysis and Comparative Mapping}

All SSR fragments experimentally mapped in P. notatum (PnEST-SSRs) were validated as derived from the specific wEST-SSR or PnSSR primer pairs and therefore were considered to be "anchored" genetic markers. Moreover, all wEST-SSR markers included the SSR motif present in the original EST although in few cases perfect repetition were detected. A BLASTn search showed that the PnESTSSRs displayed low similarity values (ranging from 40.52 to $55.27 \%$ ) with the original wheat ESTs, perhaps because of the distant genetic relationship between P. notatum and wheat within Poaceae. Nevertheless, the sequences of three amplicons matched those of the corresponding EST in a general BLASTn search. On the other hand, fragments derived from the same primer pairs gave variable degrees of similarity. Therefore, even if homologous sequences were amplified by the same primer pair, marker variation was very unlikely to be due to variability in the SSR motifs. Differences in the length of amplicons were probably associated with major sequences changes outside of the SSRs.

Because wEST-SSR markers have been used to trace chromosome segments from a wide range of grass species (Zhang et al., 2007), we performed a preliminary comparative mapping analysis by combining information from experimental and in silico mapping assays. Overall, wESTSSRs yielded multiple markers in both $P$. notatum and rice and multiple alignments in maize. Moreover, several of the putative orthologous sequences detected in maize were in agreement with the duplication of the rice segments into the maize genome described by Salse et al. (2003), confirming the alignments found in this work. A preliminary comparison of marker order and distances among $P$. notatum, rice, and maize allowed the detection of at least three putative syntenic regions. One corresponded to the chromosome segment carrying the apospory locus, with three markers having similar positions on a distal segment of rice chromosome 12 (Fig. 3). Moreover, the same group of markers was detected on maize chromosome 3 by in silico mapping. These results confirm the association of these genomic regions with apospory (Pupilli et al., 2004; Hojsgaard et al., 2011; Podio et al., 2012). The other syntenic regions corresponded to segments found on P. notatum linkage group M8a, maize chromosome 2, and rice chromosome 7 and on P. notatum linkage group M23a, maize chromosomes 2, 7, and 10, and rice chromosome 4 . Further analysis with more markers should be performed to determine whether the syntenic relationships detected in these segments are also found in other genomic regions.

Our results indicated that wEST-SSRs are transferable to Paspalum spp. and constitute a source of markers for phylogenetic analysis and mapping. Moreover, they can be used as starting point for both genetic characterization and improvement in species for which no marker technology has been developed. Wheat EST-SSR s could be used also as reference point for comparative genome analysis although orthology must be confirmed for each marker. Pasaplum notatum SSRs were useful for mapping and variability analyses in the species. Developing more of them would help improve $P$. notatum genetics and breeding. 


\section{Supplemental Information Available}

Supplemental material is available at http://www.crops. org/publications/cs.

\section{Acknowledgments}

The authors thank Ing. Florencia Galdeano for technical assistance. This study was financed by ANPCyT (Agencia Nacional de Promoción Científica y Tecnológica), Argentina, PICT 2007 No. 00476, PICT 2011 No. 1802 and PME 2006 No. 03083; CONICET (Consejo Nacional de Investigaciones Científicas y Técnicas), Argentina, PIP 11220090100613. L.A. Siena and M.E. Sartor received fellowships from CONICET. F. Espinoza, C.L. Quarin, and J.P.A. Ortiz are Career Members of CONICET.

\section{References}

Acuña, C.A., A.R. Blount, K.H. Quesenberry, K.E. Kenworthy, and W.W. Hanna. 2009. Bahiagrass tetraploid germplasm: Reproductive and agronomic characterization of segregating progeny. Crop Sci. 49:581-588. doi:10.2135/cropsci2008.07.0402

Ahn, S., and S.D. Tanksley. 1993. Comparative linkage maps of the rice and maize genomes. Proc. Natl. Acad. Sci. USA 90:79807984. doi:10.1073/pnas.90.17.7980

Altschul, S.F., W. Gish, W. Miller, E.W. Myers, and D.J. Lipman. 1990. Basic local alignment search tool. J. Mol. Biol. 215:403-410.

Barakat, A., K. Szick-Miranda, I.F. Chang, R. Guyot, G. Blanc, R. Cooke, M. Delseny, and J. Bailey-Serres. 2001. The organization of cytoplasmic ribosomal protein genes in the Arabidopsis genome. Plant Physiol. 127:398-415. doi:10.1104/pp.010265

Burson, B.L. 1978. Genome relations between Paspalum conspersum and two diploid Paspalum species. Can. J. Genet. Cytol. 20:365-372.

Burson, B.L. 1983. Phylogenetic investigations of Paspalum dilatatum and related species. In: J.A. Smith and V.W. Hays, editors, Proceedings of the XIV International. Grassland Congress, Lexington, KY. 15-24 June 1981. Westview Press, Boulder, CO. p. 170-173.

Burson, B.L. 1991a. Genome relationships between tetraploid and hexaploid biotypes of dallisgrass, Paspalum dilatatum. Bot. Gaz. 152:219-223. doi:10.1086/337883

Burson, B.L. 1991b. Homolgy of chromosomes of the X genomes in common and Uruguayan dallisgrass, Paspalum dilatatum. Genome 34:950-953. doi:10.1139/g91-147

Burson, B.L., and H.W. Bennett. 1971. Chromosome numbers, microsporogenesis, and mode of reproduction of seven Paspalum species. Crop Sci. 11:292-294. doi:10.2135/cropsci1971. 0011183X001100020038x

Burson, B.L., and H.W. Bennett. 1972. Cytogenetics of Paspalum urvillei $\times$ P. jurgensii and $P$. urvillei $\times P$. vaginatum hybrids. Crop Sci. 12:105-108. doi:10.2135/cropsci1972.0011183X001200010037x

Burson, B.L., H. Lee, and H.W. Bennett. 1973. Genome relations between tetraploid Paspalum dilatatum and four diploid Paspalum species. Crop Sci. 13:739-743. doi:10.2135/cropsci1973. 0011183X001300060045x

Burton, G.W. 1940. A cytological study of some species in the genus Paspalum. J. Agric. Res. 60:193-198.

Caponio, I., and C.L. Quarin. 1987. El sistema genético de Paspalum simplex y de un híbrido interespecífico con $P$. dilatatum. (In Spanish, with English abstract.) Kurtziana 19:35-45.
Caponio, I., and C.L. Quarin. 1990. Intra- and interspecific hybridization between Dallisgrass and Vaseygrass. Crop Sci. 30:362364. doi:10.2135/cropsci1990.0011183X003000020023x

Chase, A. 1929. North American species of Paspalum. Cont. US Natl. Herb. (28): Part 1.

Cidade, F.W., T.T. Souza-Chies, L.A.R. Batista, M. Dall'agnol, M.I. Zucchi, L. Jungmann, and A.P. Souza. 2009. Isolation and characterization of microsatellite loci in Paspalum notatum Flüggé (Poaceae). Conserv. Genet. 10:1977-1980. doi:10.1007/ s10592-009-9872-6

Cidade, F.W., T.T. Souza-Chies, F.H.D. Souza, L.A.R. Batista, M. Dall'angol, J.F.M Valls, M.I. Zucchi, and P. Souza. 2010. Microsatellite loci for Paspalum atratum (Poaceae) and cross-amplification in other species. Am. J. Bot. 97:e107-110. doi:10.3732/ ajb.1000207

Cochrane, F.C., L.B. Davin, and N.G. Lewis. 2004. The Arabidopsis phenylalanine ammonia lyase gene family: Kinetic characterization of the four PAL isoforms. Phytochemistry 66:15571564. doi:10.1016/j.phytochem.2004.05.006

Devos, K.M., and M.D. Gale. 2000. Genome relationships: The grass model in current research. Plant Cell 12:637-646.

Dice, L.R. 1945. Measures of the amount of ecologic associations between species. Ecol. 26:297-302. doi:10.2307/1932409

Don, R.H., P.T. Cox, B.J. Wainwright, K. Baker, and J.S. Mattick. 1991. 'Touchdown' PCR to circumvent spurious priming during gene amplification. Nucleic Acids Res. 19:4008. doi:10.1093/nar/19.14.4008

Espinoza, F., M.H. Urbani, E.J. Martínez, and C.L. Quarin. 2001. The breeding system of three Paspalum species with forage potential. Trop. Grassl. 35:211-217.

Essi, L., and T.T. Souza-Chies. 2007. Phylogeny of Linearia and Notata groups of Paspalum L. (Poaceae, Panicoideae, Paniceae) and related species. Genet. Resour. Crop Evol. 54:779-791. doi:10.1007/s10722-006-9148-7

Gates, R.N., C.L. Quarin, and C.G.S. Pedreira. 2004. Bahiagrass. In: L.E. Moser, B.L. Burson, and L.E. Sollenberger, editors, Warmseason (C4) grasses. ASA, CSSA, and SSSA, Madison. WI. p. 651-680.

Gene Codes. 2011. Sequencher version 4.10.1 sequence analysis software. Gene Codes Corporation, Ann Arbor, MI. http:// genecodes.com (accessed 6 Dec. 2011).

Giussani, L.M., F.O. Zuloaga, C.L. Quarin, H. Cota-Sánchez, K. Ubayasena, and O. Morrone. 2009. Phylogenetic relationships in the genus Paspalum (Poaceae: Panicoideae: Paniceae): An assessment of the Quadrifaria and Virgata informal groups. Syst. Bot. 34:32-43. doi:10.1600/036364409787602258

Hojsgaard, D.H., E.J. Martínez, C.A. Acuña, C.L. Quarin, and F. Pupilli. 2011. A molecular map of the apomixis-control locus in Paspalum procurrens and its comparative analysis with other species of Paspalum. Theor. Appl. Genet. 123:959-971. doi:10.1007/s00122-011-1639-z

Jarret, R.L., Z.W. Liu, and R.W. Webster. 1998. Genetic diversity among Paspalum spp. as determined by RFLPs. Euphytica 104:119-125. doi:10.1023/A:1018686003307

Kantety, R.V., M. La Rota, D.E. Matthews, and M.E. Sorrells. 2002. Data mining for simple sequence repeats in expressed sequence tags from barley, maize, rice, sorghum and wheat. Plant Mol. Biol. 48:501-551. doi:10.1023/A:1014875206165

Kosambi, D.D. 1944. The estimation of map distance from recombination values. Ann. Eugen. 12:172-175.

Lander, E.S., P. Green, J. Abrahamson, A. Barlow, M.J. Daly, S.E. Lincoln, and L. Newburg. 1987. MAPMAKER: An interactive 
computer package for constructing primary genetic linkage maps of experimental and natural populations. Genomics 1:174-181. doi:10.1016/0888-7543(87)90010-3

Liu, Z.W., R.L. Jarret, S. Kresovich, and R.R. Duncan. 1995. Characterization and analysis of simple sequence repeat (SSR) loci in seashore paspalum (Paspalum vaginatum Swartz). Theor. Appl. Genet. 91:47-52.

Martínez, E.J., E. Hopp, J. Stein, J.P.A. Ortiz, and C.L. Quarin. 2003. Genetic characterization of apospory in tetraploid Paspalum notatum based on the identification of linked molecular markers. Mol. Breed. 12:319-327. doi:10.1023/ B:MOLB.0000006868.12568.32

Millot, J.C. 1977. Sexuality in Paspalum dilatatum Poir ssp. dilatatum Roseng. In: M.E. Drets, N. Brum-Zorrilla, and G.A. Folle, editors, Aspects of the chromosome organization and function: Joint seminar and workshop: III Latin-American Congress of Genetics, Montevideo, Uruguay. 2-12 Feb. 1977. Asociación Lationamericana de Genética, Montevideo, Uruguay. p. 252.

Miz, R.G., and T.T. Souza-Chies. 2006. Genetic relationships and variation among biotypes of dallisgrass (Paspalum dilatatum Poir.) and related species using random amplified polymorphic DNA markers. Genet. Resour. Crop Evol. 53:541-552. doi:10.1007/s10722-005-1290-0

Oliveira, R.C., and J.F.M. Valls. 2008. Novos sinônimos e ocorrências em Paspalum L. (Poaceae). (New synonyms and occurrences for Paspalum L. (Poaceae).) (In Portuguese, with English abstract.) Hoehnea 35:289-295. doi:10.1590/S223689062008000200010

Podio, M., M.P. Rodriguez, S. Felitti, J. Stein, E.J. Martínez, L.A. Siena, C.L. Quarin, S.C. Pessino, and J.P.A. Ortiz. 2012. Sequence characterization, in silico mapping and cytosine methylation analysis of markers linked to apospory in Paspalum notatum. Genet. Mol. Biol. 35:827-837. doi:10.1590/S141547572012005000070

Powell, W., G.C. Machray, and J. Provan. 1996. Polymorphism revealed by simple sequence repeats. Trends Plant Sci. 1:215-222.

Pupilli, F., E.J. Martinez, A. Busti, O. Calderini, C.L. Quarin, and S. Arcioni. 2004. Comparative mapping reveals partial conservation of synteny at the apomixis locus in Paspalum spp. Mol. Genet. Genomics 270:539-548. doi:10.1007/s00438-0030949-5

Quarin, C.L. 1992. The nature of apomixis and its origin in Panicoid grasses. Apomixis Newsl. 5:8-15.

Quarin, C.L., and E.P. Lombardo. 1986. Niveles de ploidía y distribución geográfica de Paspalum quadrifarium (Gramineae). (In Spanish.) Mendeliana 7:101-107.

Quarin, C.L., and G.A. Norrmann. 1990. Interspecific hybrids between five Paspalum species. Bot. Gaz. 151:366-369. doi:10.1086/337837

Ritter, E., C. Gebhardt, and F. Salamini. 1990. Estimation of recombination frequencies and construction of RFLP linkage maps in plants from crosses between heterozygous parents. Genetics 125:645-654.

Rohlf, F.J. 1997. NTSYSpc: Numerical taxonomy and multivariate analysis system, version 2.02. Exeter Software, Setauket, NY.

Rua, G.H., P.R. Speranza, M. Vaio, and M. Arakaki. 2010. A phylogenetic analysis of the genus Paspalum (Poaceae) based on
cpDNA and morphology. Plant Syt. Evol. 288:227-243.

Salse, J., B. Piégu, R. Cooke, and M. Delseney. 2003. New in silico insight into the synteny between rice (Oryza sativa L.) and maize (Zea mays L.) highlights reshuffling and identifies new duplications in the rice genome. Plant J. 38:396-409. doi:10.1111/j.1365-313X.2004.02058.x

Sneath, P.H.A., and R.R. Sokal. 1973. Numerical taxonomy. Freeman and Company, San Francisco, CA.

Souza-Chies, L.E., G.H. Rua, J.F.M. Valls, B. Rogéria, and R.B. Miz. 2006. A preliminary approach to the phylogeny of the genus Paspalum (Poaceae). Genetica (The Hague) 126:15-32. doi:10.1007/s10709-005-1428-1

Speranza, P., and M. Malosetti. 2007. Nuclear and cytoplasmic microsatellite markers for the species of the Dilatata group of Paspalum (Poaceae). Plant Genet. Resour. 5:14-26. doi:10.1017/ S1479262107192145

Stein, J., S.C. Pessino, E.J. Martínez, M.R. Rodriguez, L.A. Siena, C.L. Quarin, and J.P.A. Ortiz. 2007. A genetic map of tetraploid Paspalum notatum Flügge (bahiagrass) based on singledose molecular markers. Mol. Breed. 20:153-166. doi:10.1007/ s11032-007-9083-0

Stein, J., C.L. Quarin, E.J. Martínez, S.C. Pessino, and J.P.A. Ortiz. 2004. Tetraploid races of Paspalum notatum show polysomic inheritance and preferential chromosome pairing around the apospory-controlling locus. Theor. Appl. Genet. 109:186-191. doi:10.1007/s00122-004-1614-z

Van Ooijen, J.W., and R.E. Voorrips. 2001. JoinMap3.0, Software for the calculation of genetic linkage maps. Plant Research International, Wageningen, the Netherlands.

Varshney, R.K., A. Graner, and M.E. Sorrells. 2005. Genic microsatellite markers in plants: Features and applications. Trends Biotechnol. 23:48-55. doi:10.1016/j.tibtech.2004.11.005

Yap, I.V., and R.J. Nelson. 1996. WinBoot: A program for performing bootstrap analysis of binary data to determine the confidence limits of UPGMA-based dendrograms. International Rice Research Institute, Manila, Philippines.

Yu, J.-K., T.M. Dake, S. Singh, D. Benscher, W. Li, B. Gill, and M.E. Sorrells. 2004a. Development and mapping of ESTderived simple sequence repeat markers for hexaploid wheat. Genome 47:805-818. doi:10.1139/g04-057

Yu, J.-K., M. La Rota, R.V. Kantety, and M.E. Sorrells. 2004 b. EST derived SSR markers for comparative mapping in wheat and rice. Mol. Genet. Genomics 271:742-751. doi:10.1007/ s00438-004-1027-3

Zhang, L.Y., M. Bernard, P. Leroy, C. Feuillet, and P. Sourdille. 2005. High transferability of bread wheat EST-derived SSRs to other cereals. Theor. Appl. Genet. 111:677-687. doi:10.1007/ s00122-005-2041-5

Zhang, L.Y., M. Bernard, C. Ravel, F. Balfourier, P. Leroy, C. Feuillet, and P. Sourdille. 2007. Wheat EST-SSRs for tracing chromosome segments from a wide range of grass species. Plant Breed. 126:251-258. doi:10.1111/j.1439-0523.2007.01293.x

Zuloaga, F.O., and O. Morrone. 2005. Revisión de las especies de Paspalum para América del Sur Austral (Argentina, Bolivia, sur de Brasil, Chile, Paraguay y Uruguay). (In Spanish, with English abstract.) Monographs in Systematic Botany from the Missouri Botanical Garden 102:1-297. 